\title{
Non-Binding Voluntary Agreements
}

\author{
Matthieu Glachant ${ }^{1}$, CERNA, Ecole des Mines de Paris
}

February 16, 2007

${ }^{1}$ Correspondence: 60, Boulevard St Michel, 75006 Paris, France. E-mail: glachant@ensmp.fr. Ph: + 33140519229. Fax: 33140519145 


\begin{abstract}
In reality, most voluntary agreements with polluters (VAs) are not enforceable in the sense that no legal tools are available to enforce firms' commitments. We examine whether such VAs are able to achieve an efficient level of environmental protection when they are obtained under the legislative threat of a pollution quota. We show that they can improve social welfare relative to legislative intervention when lobbying Congress is very effective and when the polluter and the regulator do not discount future costs and benefits heavily. These findings suggest that VAs should be used selectively, taking into account sector characteristics and the degree of influence of lobbying on Congress.
\end{abstract}

Keywords: environmental policy, voluntary agreements, bargaining, lobbying, enforcement.

JEL classification: D72, Q28 


\section{Introduction}

In environmental policy, a Voluntary Agreement (VA hereafter), whereby polluting firms voluntarily commit to control pollution, has become a major policy innovation of the last decade. While the use of VAs was limited initially to a few countries (e.g., Germany, Japan), they are now used extensively around the world, particularly to deal with industrial greenhouse gas emissions and waste. The use of the term "voluntary" has long been disputed since many agreements are in fact obtained under the threat of an alternative legislative intervention. The present paper focuses on such agreements preempting legislation.

In most countries, VAs are not binding. As a result, no legal tools are available to enforce firms' commitments. For instance, this is the case in Canada, France, Germany and the United States. To the best of our knowledge, the only exceptions are the UK Climate Change agreements and the Dutch "covenants" which are made enforceable through their connection with mandatory regulation. A few countries (e.g., Belgium) have tried to set up a legal framework to promote enforceable agreements with varying results essentially because most companies are reluctant to enter into binding schemes.

Non-enforceability contributes to a widespread suspicion among observers about VAs ability to genuinely improve the environment. However, this property does not imply that compliance incentives are completely lacking. The legislative threat which initiates the voluntary commitment of polluters also promotes compliance ex post since the parties to the VA contract are all aware that, in case of noncompliance, the threat will be acted upon. But non-compliance cannot be observed immediately after the contract comes into force. Furthermore, once it is discov- 
ered, enacting a new legislation takes time. In the end, several years necessarily elapse before the non-complying polluters bear the cost of the legislation. This obviously creates adverse incentives. In particular, firms may enter strategically into voluntary agreements without any willingness to comply, just to postpone legislative intervention.

In this paper, we develop a model of a non-enforceable agreement between a polluter and a benevolent regulator to address these issues. We examine whether this type of VA can lead to an efficient level of pollution abatement and how this level compares to both the first-best level and the level that might have been imposed legislatively. We make two crucial assumptions. First, the legislative threat is determined endogenously. More specifically, we explicitly model a legislative process in which the polluter lobbies a Congress, thereby reducing the stringency of mandated abatement. Otherwise, a benevolent regulator would have absolutely no reason to use a VA since it could impose the first-best legislation directly. Second, we assume that the regulator can only punish a non-complying polluter by implementing the threat in the future. In this context, the polluter's propensity to comply is driven by the endogenous stringency of the legislative quota and by the rate at which he discounts the cost of future legislation.

We do not know of any previous contribution dealing with non-enforceable $\mathrm{VAs}^{1}$. Some work in this field has explored the role of legislative threats in triggering voluntary abatement $[4,9,10,14]^{2}$. But they all assume perfect compliance. This obviously influences the analysis and the results obtained. In particular, polluters do not enter into perfectly enforceable VAs to delay legislative intervention as in our case.

Amacher and Malik [1] or Arguedas [2] do not specifically deal with VAs but 
address related issues. They examine bargaining between a polluter and a regulator over the value of an emission standard. In contrast with the papers on VAs previously mentioned, they do not assume perfect compliance. But in contrast with ours, the standard is enforceable, albeit imperfectly. In fact, they deal with the negotiation taking place during the process of setting traditional mandatory emission standards. In this context, they analyze a situation in which the regulator is ready to accept a more lenient standard if it leads the polluter to adopt an abatement technology which reduces enforcement costs. In our setting, the regulator's gain is totally different. It enters into the VA in order to avoid a politically distorted legislative quota.

The paper is organized as follows. Section 2 provides an overview of real-world voluntary agreements. Section 3 introduces the model. Section 4 identifies the circumstance under which a non-enforceable VA can emerge in equilibrium. The analysis rests on the key property that entering into a VA is a dominant strategy for the polluter. Indeed, either the Polluter enters into the VA to postpone legislative intervention without any intention to abate pollution, or it does so to comply with its commitments because the discounted cost of the legislation is sufficiently high. Accordingly, Section 5 focuses on the regulator's motives to rely on VAs. We show that the VA is more efficient than legislation in cases where lobbying Congress is very effective and when polluters and the regulator do not discount the future heavily. In Section 6, we discuss the robustness of these results and present an extension of the model in which the Polluter competes with a green lobby group to influence the Congress.

In Section 7, we conclude and discuss policy implications, particularly for climate change policies where VAs are widespread. The key lesson is that non- 
enforceable voluntary schemes are weak instruments that are potentially useful when political constraints are severe. This is probably the case when regulators seek to cut carbon emissions of energy-intensive industries.

\section{VAs in practice}

This section offers an insight into real-world voluntary agreements. It aims to identify key properties which should be incorporated into a relevant model of VAs. It rests mainly on case studies of real-world voluntary agreements presented in a recent OECD report [13] and in the book by Morgenstern and Pizer [11] who deal more specifically with voluntary schemes in the field of climate change.

In every VA, a firm or a group of firms agree to make environmental efforts beyond regulatory compliance. But the design of these voluntary commitments varies significantly. A usual classification distinguishes three broad categories [13]. Each type ultimately differs with respect to the degree of involvement of the regulator. Under public voluntary programs, the firms agree to make abatement efforts to meet goals which are established by the regulator. This is the most common form of VA in the USA. The 33/50 Program aiming at reducing the release of toxic substances is a well known example [6]. In the case of a negotiated agreement, the firms and the regulator jointly devise the commitments through bargaining. This type of VA is frequently used in Europe. As an illustration, the European Commission secured negotiated agreements during the 1990s with European (ACEA), Japanese (JAMA) and Korean (KAMA) car manufacturers to reduce new car CO2 emissions.

Under self-regulation or unilateral commitments, the Polluter takes the initia- 
tive. He freely sets up a program of environmental actions without any formal influence from public authorities. A good example is the Responsible Care initiative undertaken by the International Council of Chemical Associations which is run in 52 countries. In section 6, we consider different allocations of bargaining power between the regulator and the polluters.

The efficiency of the level of environmental protection achieved by VAs is a major practical concern. Two features are of particular concern to some observers: that VAs are voluntary, suggesting little abatement effort, and that VAs are mostly not enforceable, causing concerns about compliance.

As argued in the introduction, the fact that most agreements are developed in the face of a threat of regulation ${ }^{3}$ partly mitigates these concerns. Despite this threat, the environmental outcomes of actual VAs are arguably modest. According to the OECD report, "there are only a few cases where such approaches have been found to contribute to environmental improvements significantly different from what would have happened anyway" [13]. In the book by Morgenstern and Pizer [11], where 7 climate change VAs are assessed, conclusions are slightly more optimistic. Most estimates of the environmental effect beyond business-as-usual are in the $5-10 \%$ range.

The modest impact of many VAs suggests that legislative threats are typically weak. This weakness is due to the existence of political constraints impeding legislative action. An illuminating example is provided by the climate change VAs adopted in most EU countries in the mid-nineties. In 1994, just before the adoption of these VAs, an EU carbon tax project had been withdrawn under the pressure of lobby groups representing European energy-intensive industries. All parties were thus aware that legislation was not an easy alternative path. But, the on-going 
discussions at the international level - in which the European Union was playing a leading role and which led to the adoption of the Kyoto Protocol in 1997 - was also making clear that mandatory intervention would take place if the industry did not commit to cut emissions of greenhouse gases.

This section has attempted to highlight three key points associated with VAs, which are developed more fully in the model in the following sections. First, firms' participation in VAs and compliance are frequently driven by legislative threats. Second, lobbying usually lessens the strictness and the credibility of these threats. ${ }^{4}$ Finally, most VAs are not enforceable.

\section{The model}

We depict a policy game with three players: a benevolent Regulator, a firm (which we call the Polluter) and a Congress responsible for enacting legislation. In the first stage, the Regulator and the Polluter negotiate a Voluntary Agreement specifying a level of pollution abatement $B$ to be met by the Polluter. In case of persisting disagreement, the Regulator can ask the Congress to enact legislation. What makes the problem non-trivial is the existence of lobbying in the Congress which prevents the enactment of the socially efficient mandatory policy. In this context, the regulator must choose between two evils: either a piece of legislation distorted by lobbying or a non-enforceable VA.

In reality, certain VAs involve a coalition of polluters represented by an industry association. In our setting, the Polluter can either be a single firm or an industry. In the latter case, we assume that the members of the coalition have solved their collective action problem. 
We now enter into the details of the model. Abating pollution entails a cost borne by the Polluter which is described by an increasing and convex function $C(B)$, with $C^{\prime}(0)<1$ and $C(0)=0$. We do not grant any cost advantage to the VA: abatement costs are the same under the VA and the legislative quota.

Abatement also yields an environmental benefit. We assume that this benefit equates with the abatement level $B$, so that social welfare can be written as

$$
W(B) \equiv B-C(B)
$$

The linearity of the benefit function simplifies the notations without altering any results. Under these hypotheses, the abatement level $B^{*}$, which maximizes social welfare, solves:

$$
C^{\prime}\left(B^{*}\right) \equiv 1
$$

If the Regulator and the Polluter fail to agree, a piece of legislation mandating a level of abatement $L$ is implemented. In contrast to the VA, we assume that the Polluter perfectly complies with the quota ${ }^{5}$. The abatement quota $L$ is the outcome of a legislative process initiated by the Regulator. This process is subject to lobbying which is modelled using the approach popularized by Grossman and Helpman (see [5], chapters 7-8). We assume that the Polluter is the only lobby group exerting an influence in the Congress by making campaign contributions to a median legislator. In section 5, we analyze a variant where the polluter competes with a green lobby group.

Contributions can be in kind - by working for the legislators, by communicating, or by convincing citizens - or in cash. The Legislator maximizes his probability of re-election facing an implicit challenger by maximizing a weighted sum of the 
campaign contributions and social welfare. In fact, the Legislator is imagined as a democratically elected legislator who, during a term in Congress, collects campaign contributions he will use in a later, unmodelled, election. In this situation, he is facing a trade-off between (i) higher campaign contributions that help him to convince undecided or uninformed voters but at the cost of distorting policy choices in favor of the contributing group and (ii) a higher social welfare which increases the probability of re-election, given that voters take their welfare into consideration in their choice of candidate. Formally, his utility function is

$$
V(L, x)=\lambda W(L)+(1-\lambda) x
$$

where $L$ is the legislative quota, $x$ the campaign contribution offered to the Legislator and $\lambda \in[0,1]$, the exogenously given weight that the Legislator places on social welfare relative to the campaign contribution. One can interpret $\lambda$ as reflecting the responsiveness of the Congress to lobbying.

The timing of the legislative subgame is as follows:

1. The Regulator initiates the legislative process by asking Congress to mandate an abatement quota.

2. The Polluter offers the median Legislator a campaign contribution schedule $x(L)$ which is contingent on the adopted legislative quota $L$; this offer is assumed to be binding.

3. Then, the Legislator proposes and ratifies the quota $L$ and receives from the Polluter the contribution associated with the policy selected.

Note that, in this political procedure, the Regulator does not set the agenda of 
the Congress. It can not stipulate a particular abatement level to be voted on; instead, it requests that Congress enacts legislation. If the Regulator could stipulate an abatement level, it would propose the first-best quota $B^{*}$. Since $V(L, x) \geq 0$ for any $L$ and $x$, this quota would be approved by Congress. Thus, the political distortions described above would be circumvented.

If a VA is adopted, but the Polluter chooses not to comply, we suppose that the Regulator initiates the legislative process leading to the quota $L$. As this takes place in a future period, the Polluter discounts the cost of the sanction. Hence, he complies only if the cost of meeting the target $B$ is less than the discounted cost of the sanction:

$$
C(B) \leq \delta[C(L)+x(L)]
$$

where $\delta$ is a multiplicative discount factor $\delta \in(0,1)$ reflecting the Polluter's patience $^{6}$.

We assume that the Regulator does not observe the Polluter's discount factor, and hence is not perfectly informed about the Polluter's propensity to comply with the VA:

Assumption $1 \delta$ is a random variable whose realization is only known to the Polluter when the game begins, but whose distribution is common knowledge. The distribution of $\delta$ is uniform over the interval $[\bar{\delta}-\sigma, \bar{\delta}+\sigma] \subset[0,1] .{ }^{7}$

Introducing uncertainty of compliance can be justified on two grounds. First, it is realistic. The cost of waiting is specific to each Polluter or industry as it depends on the weight of irreversible investments, the firm's financial structure and similar idiosyncratic features. ${ }^{8}$ Second, the assumption is justified on theoretical grounds: if the Regulator knew $\delta$, the outcome of the game would entail a corner solution. 
If $\delta$ exceeded a certain threshold, the Polluter would perfectly comply with the VA; if $\delta$ fell below that threshold, the Polluter would not comply at all, and the Regulator would never use a VA.

Assumption 1 implies that the Regulator only knows the compliance probability, denoted $p(B)$, at the beginning of the game. Formally, given the distribution properties, the probability function is

$$
\begin{aligned}
p(B)= & \operatorname{Pr}(C(B) \leq \delta[C(L)+x(L)]) \\
= & \left\{\begin{array}{lll}
1, & \text { if } \quad B \leq B^{\min } \\
\frac{1}{2 \sigma}\left(\bar{\delta}+\sigma-\frac{C(B)}{C(L)+x(L)}\right), & \text { if } & B^{\min }<B<B^{\max } \\
0, & \text { if } \quad B \geq B^{\max }
\end{array}\right.
\end{aligned}
$$

where $B^{\min }$ and $B^{\max }$ denote the abatement levels such that

$$
\begin{aligned}
C\left(B^{\min }\right) & \equiv(\bar{\delta}-\sigma)(C(L)+x(L)) \\
C\left(B^{\max }\right) & \equiv(\bar{\delta}+\sigma)(C(L)+x(L)) .
\end{aligned}
$$

Finally, we assume that the Regulator also discounts the social benefit of future legislation, using the weight $\varepsilon$, which is positive but less than one.

Figure 1 shows the decision tree of the model.

Figure 1 about here 


\section{Conditions for the existence of a VA}

We begin the analysis by identifying the conditions under which an agreement between the Polluter and the Regulator is feasible. Note that any feasible agreement necessarily improves social welfare relative to legislation since it satisfies the participation constraint of the welfare-maximizing Regulator.

\subsection{The legislative subgame}

We first characterize the legislation which emerges in equilibrium. Recall that the median Legislator's utility is $V(L, x)=\lambda W(L)+(1-\lambda) x=\lambda[L-C(L)]+(1-\lambda) x$. Any feasible contribution must leave him with at least the same utility under the policy $L$ as he would achieve with no contribution. Otherwise, he would reject the offer and implement $B^{*}$ (his ideal policy when $x=0$ ). Thus, for a contribution to be feasible, we require $V(L, x) \geq V\left(B^{*}, 0\right)=\lambda\left[B^{*}-C\left(B^{*}\right)\right]$. The Polluter offers a contribution that minimizes his disutility, $C(L)+x$, subject to the feasibility constraint. Since his disutility is increasing in $x$, the feasibility constraint will bind. This contribution is implicitly defined by $V(L, x)=V\left(B^{*}, 0\right)$. Thus, the campaign contribution will depend on the quota as follows:

$$
x(L)=\frac{\lambda}{1-\lambda}\left[W\left(B^{*}\right)-W(L)\right]
$$

In light of eqs (1) and (6), the Polluter minimizes

$$
C(L)+x(L)=\frac{\lambda W\left(B^{*}\right)-\lambda L+C(L)}{1-\lambda}
$$

As the function (7) is convex, there is a unique value $L^{*}$ that minimizes the 
Polluter's disutility, where $C^{\prime}\left(L^{*}\right)=\lambda$. The Polluter offers the campaign contribution $x\left(L^{*}\right)$ in exchange for adoption of the quota $L^{*}$. We collect these findings in

Lemma 1 The equilibrium legislation $L^{*}$ is such that $C^{\prime}\left(L^{*}\right)=\lambda$ while the equilibrium campaign contribution is $x\left(L^{*}\right)=\frac{\lambda}{1-\lambda}\left[W\left(B^{*}\right)-W\left(L^{*}\right)\right]$.

Since $\lambda<1$, it follows that $L^{*}<B^{*}$.

\subsection{The VA subgame}

We turn next to the analysis of the VA. When negotiating over the level of voluntary abatement $B$, the Polluter's utility obviously depends on his compliance decision. Given the compliance condition (4), his payoff is thus given by

$$
\max \left\{-C(B),-\delta\left[C\left(L^{*}\right)+x\left(L^{*}\right)\right]\right\}
$$

It follows that entering into a VA is a dominant strategy for the Polluter since

Lemma 2 The Polluter's payoff under a VA is higher than his legislative payoff for any level of voluntary abatement.

Proof. Recall that legislative payoff is simply $-C\left(L^{*}\right)-x\left(L^{*}\right)$. If $C(B) \leq$ $\delta\left[C\left(L^{*}\right)+x\left(L^{*}\right)\right]$, then $-C(B) \geq-\left[C\left(L^{*}\right)+x\left(L^{*}\right)\right]$ since $\delta<1$. Alternatively, if $C(B)>\delta\left[C\left(L^{*}\right)+x\left(L^{*}\right)\right]$, we obviously have $-\delta\left[C\left(L^{*}\right)+x\left(L^{*}\right)\right]>-\left[C\left(L^{*}\right)+x\left(L^{*}\right)\right]$.

The intuition of the lemma is simple. The Polluter is willing to participate in any VA because discounting makes the sanction cost $\delta\left[C\left(L^{*}\right)+x\left(L^{*}\right)\right]$ strictly less 
than his disagreement disutility $C\left(L^{*}\right)+x\left(L^{*}\right)$. As a result, the Polluter enters into a VA either because it is less costly than legislation (when $B$ is low), or because he anticipates non-compliance (when $B$ is higher).

This property greatly simplifies the analysis: non-enforceable VAs are only driven by the preferences of the Regulator.

We now define the Regulator's payoff under the VA route:

$$
W^{V A}(B) \equiv p(B) W(B)+[1-p(B)] \varepsilon W\left(L^{*}\right)
$$

where $\varepsilon$ is the rate at which it discounts the social benefit of future legislation in case of non-compliance. Note that, as is usual in the political economy literature, we assume that the Regulator does not care about the campaign contribution as it is a transfer between the Polluter and the Congress. The alternative assumption that the contribution is a cost included in the welfare function would not reverse the results. It would simply make a VA more likely by creating an additional incentive for the Regulator to use this instrument.

Assumption 1 introduces (one-sided) asymmetric information in the game. In this case, bargaining theory tells us that satisfying players' participation constraints may not be sufficient to ensure the existence of ex post efficient bargaining outcomes when payoffs are correlated (see [12] for a general discussion) $)^{9}$. Intuitively, this is so because the informed player has an incentive to manipulate the information he transmits to the uninformed player. More precisely, he has an incentive to pretend he will comply with the VA. As the Regulator is aware of this 'incentive to lie', the minimal level of abatement it might be willing to accept may be strictly higher than the reservation level of the 'high type' Polluter who 
complies with the VA. However, this general argument does not apply to our case since the Regulator is aware that the polluter is willing to accept any VA (see Lemma 2). We establish the argument more rigorously in

Lemma 3 If there exists a level of abatement $B$ such that $W^{V A}(B)>W\left(L^{*}\right)$, then there exists a bargaining procedure such that bargaining yields an ex-post efficient Bayes Nash equilibrium.

Proof. Consider the following bargaining procedure. The Polluter makes an offer to the Regulator. If the offer is accepted, the agreement is struck and the game ends. But if the Regulator rejects the offer, then the game ends with no agreement. Letting $\tilde{B}(\delta)$ denoting the Polluter's offer when his type is $\delta$, the following set of strategies is a Bayes Nash equilibrium: $\forall \delta \in[\bar{\delta}-\sigma, \bar{\delta}+\sigma], \tilde{B}(\delta)=B^{\circ}$ such that $W^{V A}\left(B^{\circ}\right)=W\left(L^{*}\right)$; and the Regulator accepts the offer. The outcome is obviously Pareto efficient, because any deviation from $B^{\circ}$ makes one player worse off. This bargaining procedure allocates all the bargaining power to the Polluter. Under the hypothesis that the Regulator has the bargaining power, it would make an offer maximizing her payoff which will also be accepted since the Polluter agrees in every case.

\section{$5 \quad$ Regulator's bargaining payoff}

\subsection{General properties}

Lemma 3 tells us that a necessary and sufficient condition for the existence of a VA is the existence of an abatement level $B$ such that $W^{V A}(B)>W\left(L^{*}\right)$, or 
alternatively

$$
\max \left\{W^{V A}(B): B \geq 0\right\}>W\left(L^{*}\right)
$$

the highest VA welfare must exceed the equilibrium legislative welfare. In this section, we investigate the properties of $W^{V A}$ in order to identify the circumstances under which condition (10) is satisfied.

Combining (5) and (9) yields

$$
W^{V A}(B)=\left\{\begin{array}{lll}
W(B) & \text { if } & B \leq B^{\min } \\
F(B) & \text { if } & B^{\min }<B<B^{\max } \\
\varepsilon W\left(L^{*}\right) & \text { if } & B \geq B^{\max }
\end{array}\right.
$$

where

$$
F(B) \equiv \frac{1}{2 \sigma}\left(\bar{\delta}+\sigma-\frac{C(B)}{C\left(L^{*}\right)+x\left(L^{*}\right)}\right)\left[W(B)-\varepsilon W\left(L^{*}\right)\right]+\varepsilon W\left(L^{*}\right),
$$

Then, we establish a set of properties of $F$ which will be used to represent $W^{V A}$ diagrammatically.

Lemma 4 We have:

1) $F^{\prime}(0)>W^{\prime}(0)$.

2) $F(0)=0$.

3) If $W\left(B^{\max }\right)<\varepsilon W\left(L^{*}\right)$, then $F^{\prime}>0$ for any $B \in\left[B^{\min }, B^{\max }\right]$.

4) If $W\left(B^{\max }\right) \geq \varepsilon W\left(L^{*}\right)$ and $F^{\prime}\left(B^{\min }\right)>0$, then $F$ admits a unique interior maximum, denoted $\hat{B}$, over $\left[B^{\min }, B^{\max }\right]$.

5) If $W\left(B^{\max }\right) \geq \varepsilon W\left(L^{*}\right)$ and $F^{\prime}\left(B^{\min }\right) \leq 0$, then $F^{\prime} \leq 0$ for any $B \in$ $\left[B^{\min }, B^{\max }\right]$. 
Proof. See the appendix.

Using these properties, Figures $2 \mathrm{a}, 2 \mathrm{~b}$, and $2 \mathrm{c}$ show $W^{V A}$ as a function of $B$ in the different cases. In all cases, $W^{V A}(B)$ equals $W(B)$ when $B \leq B^{\text {min }}$ (since $p(B)=1)$ and $\varepsilon W\left(L^{*}\right)$ when $B \geq B^{\max }$. Between $B^{\min }$ and $B^{\max }, W^{V A}$ is either strictly decreasing (Fig. 2a), non-monotonic (Fig. 2b) or strictly increasing (Fig. 2c).

Figure 2a about here

Figure 2b here

Figure 2c about here

Looking at Fig. 2a-c, it is evident that the highest level of VA social welfare is given by

$\max \left\{W^{V A}(B): B \geq 0\right\}=\left\{\begin{array}{lll}W\left(B^{\min }\right) & \text { if } & W\left(B^{\max }\right) \geq \varepsilon W\left(L^{*}\right) \text { and } F^{\prime}\left(B^{\min }\right) \leq 0 \\ F(\hat{B}) & \text { if } & W\left(B^{\max }\right) \geq \varepsilon W\left(L^{*}\right) \text { and } F^{\prime}\left(B^{\min }\right)>0 \\ \varepsilon W\left(L^{*}\right) & \text { if } & W\left(B^{\max }\right)<\varepsilon W\left(L^{*}\right)\end{array}\right.$

Hence,

Proposition 1 1) If $W\left(B^{\max }\right) \geq \varepsilon W\left(L^{*}\right)$, a welfare-improving $V A$ exists if either

a) $F^{\prime}\left(B^{\mathrm{min}}\right) \leq 0$ and $B^{\mathrm{min}}>L^{*}$ or b) $F^{\prime}\left(B^{\mathrm{min}}\right)>0$ and $F(\hat{B})>W\left(L^{*}\right)$.

2) If $W\left(B^{\max }\right)<\varepsilon W\left(L^{*}\right)$, there never exists a VA yielding a higher welfare than the legislative quota. 
Proof. The proof is straightforward since we know $\max \left\{W^{V A}(B): B \geq 0\right\}$ in the different cases. In the particular case where $W\left(B^{\max }\right)<\varepsilon W\left(L^{*}\right)$, no VAs are feasible since $\max \left\{W^{V A}\right\}=\varepsilon W\left(L^{*}\right)$ which is strictly less than $W\left(L^{*}\right)$ (since $\varepsilon<1)$.

Proposition 1 is the main proposition of the paper. It establishes that, depending on parameters' values, we can either observe a VA or not. In addition, a VA may involve a risk of non-compliance or not. For instance, assuming that the Regulator has all the bargaining power, it selects the abatement level $\hat{B}$ maximizing $W^{V A}$ when $W\left(B^{\max }\right) \geq \varepsilon W\left(L^{*}\right)$ and $F^{\prime}\left(B^{\min }\right)>0$. And we know from (5) that $p(\hat{B})<1$. Alternatively, if $F^{\prime}\left(B^{\text {min }}\right) \leq 0$, the Regulator chooses $B^{\text {min }}$ with a compliance probability equal to 1 .

\subsection{Interpretation of Proposition 1}

Proposition 1 does not allow us to see how the different parameters influence the likelihood of a VAs existence. For instance, the condition $B^{\mathrm{min}}>L^{*}$ does not necessarily imply that $\lambda$ should be less than a certain level to obtain a VA since both $L^{*}$ and $B^{\text {min }}$ increase with $\lambda$. To further the interpretation of the model, we now investigate the properties of the equilibrium when abatement costs are quadratic, with $C(B)=\frac{1}{2} \theta B^{2} ;$ where $\theta>0 .^{10}$

Calculations included in the appendix characterize the key relationships between the conditions of Proposition 1 and the parameters $\lambda, \bar{\delta}$, and $\varepsilon$. They show that

Proposition 2 The VA outcome is closer to the first-best one than the legislative quota when the Congress is strongly responsive to lobbying (a low $\lambda$ ) and when the 
Polluter and the Regulator are patient (high $\bar{\delta}$ and $\varepsilon$ ).

The influence of the lobbying parameter $\lambda$ is not so intuitive as a high responsiveness to lobbying affects VA welfare in two contradictory ways. On the one hand, it reduces the stringency of the legislative quota $L^{*}$, thereby increasing the Regulator's interest in VAs. On the other hand, it increases the risk of noncompliance associated with VAs since the size of the sanction $\delta\left(C\left(L^{*}\right)+x\left(L^{*}\right)\right)$ directly depends on the strictness of the quota. Proposition 2 tells us that the former effect unambiguously outweighs the latter.

The result that the more patient the Polluter, the larger the scope for welfareimproving VAs is not surprising as a low discount rate mitigates the VA compliance problem by increasing the size of the sanction $\delta\left(C\left(L^{*}\right)+x\left(L^{*}\right)\right)$ borne by the Polluter. The reason for a patient Regulator's tendency to prefer VAs is also simple. Key in explaining this is the way the Regulator values non-compliance. In the case where the Polluter fails to comply, the Regulator's utility is $\varepsilon W\left(L^{*}\right)$ which corresponds to the delayed implementation of the legislative quota. This benefit obviously increases with $\varepsilon$, making the use of VAs more attractive.

\section{Robustness of the results}

The model presented here is fairly simplistic. It is worth discussing the robustness of the insights it gives and some possible extensions. Three criticisms/questions come quickly to mind: the impact of bargaining power on outcomes, the fact that there is no green lobby group acting in the Congress and the assumption that polluters have solved their collective action problem. We now consider these points. 


\subsection{Bargaining power}

When interpreting Proposition 1 in subsection 5.2, we assume that the Regulator has all the bargaining power. How do different allocations of bargaining power influence the results obtained? This question echoes the classification previously mentioned which distinguishes three categories of VAs: public voluntary programs developed by public authorities, to which companies are invited to participate, negotiated agreements between polluters and public authorities, and unilateral commitments made by polluters. In settings, like ours, where VAs are driven by Regulator's threats, this classification ultimately describes different allocations of bargaining power between the regulator and the polluters. ${ }^{11}$

Given that the Regulator seeks to maximize welfare, it is very intuitive that

Proposition 3 Social welfare associated with a non-enforceable VA increases with the Regulator's bargaining power.

Proof. See the appendix.

\subsection{Lobbying by a green group and free riding}

The fact that the Polluter is the only active lobby group in the Congress and that free riding does not hinder its lobbying efforts may pose a problem as one could expect better legislative outcomes once these assumptions are relaxed.

In this subsection, we adopt a common agency framework in which the Legislator is the agent of two principals - the Polluter and a green lobby group - both offering contributions. For the sake of tractability, we only consider VAs involving 
perfect compliance in equilibrium $\left(p\left(B^{V A}\right)=1\right)$ and we assume that the Regulator has all the bargaining power. Formally, the Legislator's utility function is now

$$
V(L, x)=\lambda W(L)+(1-\lambda)\left(x_{P}(L)+x_{G}(L)\right),
$$

where $x_{P}(L)$ and $x_{G}(L)$ are the Polluter's and the green group's contingent contribution schedules, respectively.

We also introduce free riding considerations, admittedly roughly, by assuming that the Polluter's lobbying cost is $x_{P}(L) /(1-\rho)$ for making a contribution $x_{P}(L)$ to the median legislator with $0 \leq \rho<1$. $\rho$ is a new parameter capturing the idea that, when some firms within an industry fail to cooperate, remaining contributors should make additional efforts. Note that $\rho$ is inversely related to lobbying effectiveness. Under this assumption, Polluter's legislative pay off is now

$$
-C(L)-\frac{x_{P}(L)}{1-\rho}
$$

Turning next to the green lobby group, we suppose that it is only concerned with the environmental benefit of legislation so that its utility under legislation is

$$
L-\frac{x_{G}(L)}{1-\gamma}
$$

with $0 \leq \gamma<1$. Note that, when $\gamma>\rho$, the green group is less effective in lobbying activities than the Polluter.

The derivation of the political equilibrium closely follows Grossman and Helpman [5] and is left out for ease of presentation. When the lobby groups choose their contributions, the key difference from the previous sections is that "walk- 
ing away" no longer implies that the Legislator will implement the optimal quota $B^{*}$. Rather, if a group abstains from lobbying, the Legislator implements the best legislation given the other group's contribution.

In addition, we assume that contributions schedules are globally compensating. This means that each contribution function "compensates" the group for its different evaluations of the two policy options. Accordingly, the contribution functions are given by

$$
\frac{x_{P}(L)}{1-\rho}=C\left(L^{-P}\right)-C(L) \text { and } \frac{x_{G}(L)}{1-\rho}=L-L^{-G}
$$

where $L^{-P}$ and $L^{-G}$ denote the legislative quotas when the Polluter or the green group are not involved, respectively. This assumption is routinely made in the literature because it is necessary to pin down equilibrium contributions (for detailed explanations and justifications, see [5], pp 265-270) ${ }^{12}$. Plugging (13) in the Legislator's objective function (12) and omitting constant terms, we obtain the following maximization problem

$$
\max _{L} \lambda W(L)+(1-\lambda)[(1-\gamma) L-(1-\rho) C(L)]
$$

We derive the first-order condition and solve for $L$ so that equilibrium legislation is

$$
L^{*}=\frac{1}{\theta}\left(\frac{1-\gamma(1-\lambda)}{1-\rho(1-\lambda)}\right)
$$

Note that the quota $(14)$ coincides with the first-best quota $\left(B^{*}\right)$ when lobby groups are equally effective $(\rho=\gamma)$. This obviously implies that

Proposition 4 When groups are equally effective in lobbying activities $(\rho=\gamma)$, 
VAs never dominate legislative intervention in equilibrium since the legislative quota is socially optimal.

This proposition illustrates a general feature of contribution-based lobbying games. Distortions are driven by the existence of a political asymmetry between the groups affected by the policy, either because lobby groups are not equally effective $(\rho \neq \gamma)$ or because one group is not represented in the lobbying game like in the previous sections.

By contrast, if lobbying effectiveness is heterogeneous, calculations provided in the appendix show that

Proposition 5 When $\rho \neq \gamma$, a VA yields a higher welfare than legislation when the lobbying parameter $\lambda$ is low, when the Polluter is patient (a high $\bar{\delta}$ ), when the Polluter's lobbying effectiveness is low (as reflected by a high $\rho$ ), or when the green group's lobbying effectiveness is high (as reflected by a small $\gamma$ ).

If $\lambda$ is sufficiently low - that is, if $\lambda<(1-\rho) \sqrt{\bar{\delta}-\sigma} /(1-\rho \sqrt{\bar{\delta}-\sigma})$ - the $V A$ even yields the first-best abatement level $B^{*}$.

Proof. See the appendix.

The first part of Proposition 5 is in line with previous results (the impact of $\lambda$ and $\bar{\delta}$ ). The influence of lobbying effectiveness' parameters $\rho$ and $\gamma$ is less intuitive. And the fact that voluntary abatement can be socially optimal when $\lambda$ is sufficiently low is clearly a new and striking result. In order to understand the underlying intuition of these results, recall that the Regulator' ability to implement a strict VA is constrained by the compliance condition

$$
C(B)<\delta\left(C\left(L^{*}\right)+\frac{x_{P}\left(L^{*}\right)}{1-\rho}\right)
$$


This expression makes clear that the higher the value of $\rho$, the wider the room for a strict VA with which the Polluter will comply. The reason is extremely simple. As compared to the preceding sections in which $\rho$ was set to zero, our new assumption increases the Polluter's lobbying cost $x_{P}\left(L^{*}\right) /(1-\rho)$ and thus the scope for VAs by increasing the size of compliance incentives.

Now, let us substitute (13) in (15). The compliance condition becomes

$$
C(B)<\delta C\left(L^{-P}\right)
$$

It is then clear that the room for a strict VA increases with $L^{-P}$. And $L^{-P}$ is high when the green lobby is very effective (a small $\gamma$ ) or when the Legislator is strongly responsive to lobbying (a low $\lambda$ ). If $L^{-P}$ is sufficiently high, we can perfectly observe a VA involving $B^{*}$. This occurs when $C\left(B^{*}\right)<\delta C\left(L^{-P}\right)$ as we observe perfect compliance with $B^{V A}=B^{*}$.

Therefore, this extension does not change the general message that VAs are suitable in contexts in which the responsiveness of the Congress to lobbying is high. But, in addition to this, it shows that the introduction of competition with a green lobby group or a decrease in the Polluter's lobbying effectiveness (by setting $\rho \neq 0$ ) tend to increase the scope for VAs by raising the Polluter's lobbying cost and thus his propensity to comply.

However, note that the robustness of the particular result that the VA can be socially optimal is questionable. In fact, the size of the left hand side of (15) directly depends on the assumption that the Polluter's contribution schedule is globally compensating. This assumption, though a usual and a necessary tool to derive equilibrium contributions, clearly determines a high level of contribution 
since it essentially means that the Legislator is able to extract the entire lobbying surplus from the two groups.

\section{Conclusion}

We have developed a model of non-enforceable VAs under the threat of a legislative quota with two main assumptions. The first is that the Polluter is an active lobby group in the Congress influencing the legislative process. This political distortion makes possible the entry of the Regulator into a VA which avoids the enactment of a piece of politically distorted legislation. The fact that the VA contract is non-binding is the second key assumption. As a result, the Regulator can only punish a non-complying Polluter by implementing the legislation at a later stage.

We show that a non-enforceable VA can be a better instrument than a legislative quota in specific circumstances. This is particularly the case when lobbying exerts a strong influence on the Congress. Interestingly, the result is not very intuitive because a distorted legislative process yields two opposite effects. On the one hand, it obviously reduces the strictness of the legislative quota. On the other hand, it damages VA social welfare since a lax legislative quota provides lower compliance incentives. Our analysis shows that the former effect is stronger that the latter.

This finding contradicts the recurrent policy recommendation that VAs should be developed under credible legislative or regulatory threats (for instance, see [13]). When threats are credible and sufficiently strong, we show that legislation is preferable. VAs are also shown to yield a higher social welfare than legislative quotas when the polluter and the regulator do not discount future costs and benefits 
heavily.

To conclude, the main finding of the paper is that non-binding VAs are weak instruments which are potentially useful in adverse political contexts. In practice, they are particularly widespread in climate change policies. Do our results suggest that VAs are suitable for these policies? To a large extent, answering this question is speculative as political contexts vary greatly across countries and the key parameters of the model (e.g., $\lambda$ ) are not quantifiable in a consistent and comparable way.

Nevertheless, our model pinpoints two arguments in favor of climate change VAs. First, they have mostly been developed in energy-intensive industries (steel, glass, cement, chemicals, etc.) which are typically very effective in lobbying activities. One reason being that free riding is less likely in sectors where companies are few and large and in which energy (and thus abatement) costs represent a significant share of production costs. Second, climate change is a long term policy concern for which immediate action is less crucial than mid- or long-term policy strategies. As a result, the regulators' cost of waiting is probably low in comparison with other policy areas. This promotes the adoption of VAs since a key risk associated with their use is to delay legislative intervention in the event of non-compliance.

Our model is quite simplistic and several extensions or improvements could be pursued. In our view, the two most promising lines of research are the following. First, one should try to be more specific as to the type of VAs analyzed. ${ }^{13}$ In this regard, it would be interesting to investigate specifically the case of industry-wide agreement while relaxing the assumption that firms fully overcome their free-rider difficulties. Second, the hypothesis of perfect information about abatement cost 
should be relaxed. This would probably reduce the scope for VAs since information asymmetry is particularly detrimental in bargaining contexts.

\section{Acknowledgements}

I would like to thank John Maxwell, two anonymous referees and many seminar participants at the University of Cergy-Pontoise, Université Catholique de Louvain and CIRED for their helpful comments. The usual disclaimer applies.

\section{Appendix}

\subsection{Proof of Lemma 4}

To begin with, we differentiate $F$ which leads to

$$
F^{\prime}(B)=-C^{\prime}(B) /\left((2 \sigma)\left(C\left(L^{*}\right)+x\left(L^{*}\right)\right)\right)\left[W(B)-\varepsilon W\left(L^{*}\right)\right]+p(B) W^{\prime}(B)
$$

Then we consider the different properties in turn.

1) We have $F^{\prime}(0)=\frac{1}{\sigma}\left[C^{\prime}(0) / C\left(L^{*}\right)+x\left(L^{*}\right)\right] \varepsilon W\left(L^{*}\right)+\left(\frac{\bar{\delta}+\sigma}{2 \sigma}\right)\left(1-C^{\prime}(0)\right)$. From $W^{\prime}(0)=1-C^{\prime}(0)$ follows $F^{\prime}(0)=\frac{1}{\sigma}\left[C^{\prime}(0) / C\left(L^{*}\right)+x\left(L^{*}\right)\right] \varepsilon W\left(L^{*}\right)+\left(\frac{\bar{\delta}+\sigma}{2 \sigma}\right) W^{\prime}(0)$ which is higher than $W^{\prime}(0)$ since $\frac{1}{\sigma}\left[C^{\prime}(0) / C\left(L^{*}\right)+x\left(L^{*}\right)\right] \varepsilon W\left(L^{*}\right)>0$ and $(\bar{\delta}+\sigma) / 2 \sigma \geq$ 1 (since $\bar{\delta} \geq \sigma$ by hypothesis).

2) Given (11), $F(0)=0$ is obvious.

3) If $W\left(B^{\max }\right)<\varepsilon W\left(L^{*}\right)$, then $W(B)-\varepsilon W\left(L^{*}\right)<0$ for any $B \in\left[B^{\min }, B^{\max }\right]$. Therefore, the first term of (16) is positive in the same interval. The second term is also positive since $W^{\prime}>0$ when $B<B^{*}$. Hence $F^{\prime}>0$. 
4) If $W\left(B^{\max }\right) \geq \varepsilon W\left(L^{*}\right)$, then $F$ is concave for any $B \in\left[B^{\min }, B^{\max }\right]$ since

$$
\begin{aligned}
F^{\prime \prime}(B)= & -\frac{1}{2 \sigma}\left[C^{\prime \prime}(B) / C\left(L^{*}\right)+x\left(L^{*}\right)\right]\left[W(B)-\varepsilon W\left(L^{*}\right)\right] \\
& -\frac{1}{\sigma}\left[C^{\prime}(B) /\left(C\left(L^{*}\right)+x\left(L^{*}\right)\right)\right] W^{\prime}(B)-p(B) C^{\prime \prime}(B)
\end{aligned}
$$

which is obviously negative (since $C^{\prime}(B), C^{\prime \prime}(B)$ and $W^{\prime}(B)>0$ ). In addition,

$$
F^{\prime}\left(B^{\max }\right)=-C^{\prime}\left(B^{\max }\right) /\left((2 \sigma)\left(C\left(L^{*}\right)+x\left(L^{*}\right)\right)\right)\left[W\left(B^{\max }\right)-\varepsilon W\left(L^{*}\right)\right]<0
$$

It implies that there exists a unique interior maximum defined by the first order condition $F^{\prime}(\hat{B})=0$ for any $B \in\left[B^{\min }, B^{\max }\right]$ if $F^{\prime}\left(B^{\min }\right)>0$ while:

5) $F^{\prime}(B) \leq 0$ if $F^{\prime}\left(B^{\min }\right) \geq 0$.

\subsection{Proof of Proposition 2}

We interpret first Part 1) of Proposition 1 where $W\left(B^{\max }\right) \geq \varepsilon W\left(L^{*}\right)$. Then, we consider Part 2) where $W\left(B^{\max }\right)<\varepsilon W\left(L^{*}\right)$.

\subsubsection{Case 1: $W\left(B^{\max }\right) \geq \varepsilon W\left(L^{*}\right)$}

In this case, Proposition 1 tells us that a welfare-improving VA exists if either a) $F^{\prime}\left(B^{\text {min }}\right) \leq 0$ and $B^{\text {min }}>L^{*}$ or b) $F^{\prime}\left(B^{\text {min }}\right)>0$ and $F(\hat{B})>W\left(L^{*}\right)$. Moreover, assuming that the Regulator has all the bargaining power, the equilibrium VA is $B^{V A}=B^{\text {min }}$ if $F^{\prime}\left(B^{\text {min }}\right) \leq 0$ and $B^{V A}=\hat{B}$ if $F^{\prime}\left(B^{\text {min }}\right)>0$. We now examine the influence of the parameters $\lambda, \varepsilon, \bar{\delta}$ on these results by distinguishing two subcases $L^{*}<B^{\mathrm{min}}$ and $L^{*} \geq B^{\mathrm{min}}$. 
Subcase a: $L^{*}<B^{\text {min }}$. In this case, Proposition 1 already tells us that VAs dominate legislation if $F^{\prime}\left(B^{\mathrm{min}}\right) \leq 0$. Here, we show that the same is true when $F^{\prime}\left(B^{\text {min }}\right)>0$. For ease of presentation, let $\hat{L}$ denote the value of $L$ implicitly defined by the two conditions $W(\hat{L})=F(\hat{B})$ and $\hat{L}<\hat{B}$. Using this notation, $F(\hat{B})>W\left(L^{*}\right)$ is the same as $L^{*}<\hat{L}$. Diagrammatically, Fig. 2b immediately shows that $B^{\text {min }}<\hat{B}$. Hence, $L^{*}<B^{\text {min }}$ necessarily implies that $L^{*}<\hat{L}$. Note that $L^{*}<B^{\min }$ is equivalent to $\lambda<\bar{\delta}-\sigma$.

Subcase b: $L^{*} \geq B^{\text {min }}$. In this case, the only feasible agreement is $B^{V A}=\hat{B}$ which is observed when $F^{\prime}\left(B^{\text {min }}\right)>0$ and $F(\hat{B})>W\left(L^{*}\right)$. Let $g(\lambda, \varepsilon, \bar{\delta})$ the function such that $g(\lambda, \varepsilon, \bar{\delta})=F(\hat{B})-W\left(L^{*}\right)$. We now study the properties of $g$ to identify how $\lambda, \varepsilon$, and $\bar{\delta}$ influence its sign.

It is convenient to develop $g(\lambda, \varepsilon, \bar{\delta})$ as follows

$$
g(\lambda, \varepsilon, \bar{\delta})=p(\hat{B})\left[W(\hat{B})-\varepsilon W\left(L^{*}\right)\right]-(1-\varepsilon) W\left(L^{*}\right)
$$

Differentiating (17) with respect to $\varepsilon$ and $\bar{\delta}$, substituting $F^{\prime}(\hat{B})=0$ and rearranging yields

$$
\begin{aligned}
& \frac{\partial g}{\partial \varepsilon}=[1-p(\hat{B})] W\left(L^{*}\right) \\
& \frac{\partial g}{\partial \bar{\delta}}=\frac{1}{2 \sigma}\left[W(\hat{B})-\varepsilon W\left(L^{*}\right)\right]
\end{aligned}
$$

Both derivatives are positive, meaning that rising $\varepsilon$ and/or $\bar{\delta}$ promotes the existence of welfare-improving VAs.

Turning next to the parameter $\lambda$, note that $L^{*} \geq B^{\text {min }}$ is equivalent to $\lambda \in$ 
$[\bar{\delta}-\sigma, 1]$. In the particular case where $L^{*}=B^{\min }$, or $\lambda=\bar{\delta}-\sigma, g>0$ since $\hat{B}>$ $B^{\text {min }}$ By contrast, if $\lambda=1$, or $L^{*}=B^{*}$, we have $g(1, \varepsilon, \bar{\delta})=F(\hat{B})-W\left(B^{*}\right)<0$. In between these two values of $L^{*}$, simulations available upon request confirm that $g$ is positive for small values of $\lambda$ and then becomes positive beyond a certain threshold.

\subsubsection{Case 2: $W\left(B^{\max }\right)<\varepsilon W\left(L^{*}\right)$}

In this case, the legislative quota Pareto dominates the VA . Here, we establish that, if $\lambda, \varepsilon$, and $\bar{\delta}$ are such that $W\left(B^{\max }\right)<\varepsilon W\left(L^{*}\right)$, this implies that $L^{*}>$ $\max \left\{B^{\min }, \hat{L}\right\}$. Put differently, the condition $W\left(B^{\max }\right)<\varepsilon W\left(L^{*}\right)$ is not binding and the properties of $\lambda, \varepsilon$, and $\bar{\delta}$ identified in Case 1 are sufficient to define the scope for a welfare-improving agreement.

From $W\left(B^{\max }\right)<\varepsilon W\left(L^{*}\right)$ follows $B^{\max }<L^{*}$ since $\varepsilon<1$. This directly implies $L^{*}>B^{\text {min }}$. Fig. $2 \mathrm{~b}$ also shows that $W\left(B^{\max }\right)>F(\hat{B})$. This implies that $L^{*}>\hat{L}$, or alternatively $W\left(L^{*}\right)>F(\hat{B})$, since $W\left(B^{\max }\right)<\varepsilon W\left(L^{*}\right)<W\left(L^{*}\right)$.

\subsubsection{Summary}

According to subcase 1a, there exists a welfare-improving VA if $\lambda$ and $\bar{\delta}$ are such that $\lambda<\bar{\delta}-\sigma$. If $\lambda \geq \bar{\delta}+\sigma$, the analysis of subcase 1b tells us that we have a VA if $\lambda$ is not too high and/or if $\bar{\delta}$ and $\varepsilon$ are sufficiently high. Finally, the condition of Case 2 is not binding. These elements converge to establish that VAs emerge when $\lambda$ is low and/or when $\bar{\delta}$ and $\varepsilon$ are high. 


\subsection{Proof of Proposition 4}

We obviously restrict the analysis to the case where a VA is feasible, that is when $W^{V A}\left(B^{\max }\right) \geq \varepsilon W\left(L^{*}\right)$. When the Regulator has all the bargaining power, we have

seen that $B^{V A}=\max \left\{B^{\min }, \hat{B}\right\}$ which maximizes expected welfare. Now we show that $B^{V A}=L^{*}$ in the opposite case in which the Polluter has all the bargaining power. Given (8), his first-best VA is simply $B^{V A}=0$. But this does not satisfy the Regulator's participation constraint $W^{V A}\left(B^{V A}\right) \geq W\left(L^{*}\right)$. Therefore, this condition is binding in equilibrium, that is $W^{V A}\left(B^{V A}\right)=W\left(L^{*}\right)$. Figures $2 \mathrm{~b}$ and 2c then show that this equation admits two roots: $L^{*}$ and the abatement level denoted $L^{\prime}$ such that $W^{V A}\left(B^{V A}\right)=W\left(L^{\prime}\right)$ and $L^{\prime}>\max \left\{B^{\min }, \hat{B}\right\}$. Ideally, the Polluter would choose the abatement level maximizing his payoff, that is either $L^{\prime}$ if he does not comply when $B^{V A}=L^{\prime}$ or $L^{*}$ if he does. But, the Polluter cannot select $L^{\prime}$ because, in doing so, he reveals to the Regulator that he will not comply. Hence $B^{V A}=L^{*}$ in equilibrium. Under intermediate allocations of bargaining power, $B^{V A}$ lies in between $L^{*}$ and $\max \left\{B^{\min }, \hat{B}\right\}$ and the stronger the Regulator's bargaining power, the closer $B^{V A}$ to the second best optimum $\max \left\{B^{\min }, \hat{B}\right\}$.

\subsection{Proof of Proposition 5}

We first identify the highest level of abatement $B^{\text {min }}$ below which the Polluter complies with the VA. It is implicitly defined by

$$
p\left(B^{\min }\right)=\frac{1}{2 \sigma}\left(\bar{\delta}+\sigma-\frac{C\left(B^{\min }\right)}{C\left(L^{*}\right)+x^{P}\left(L^{*}\right) /(1-\rho)}\right)=1
$$


Substituting $x^{P}\left(L^{*}\right) /(1-\rho)=C\left(L^{-P}\right)-C\left(L^{*}\right)$ in this equation and rearranging yields

$$
C\left(B^{\min }\right)=(\bar{\delta}-\sigma) C\left(L^{-P}\right)
$$

$L^{-P}$ is the equilibrium of the single-lobby game in which the contribution $x_{G}(L)$ is still given by (6) while the green group maximizes $L-x_{G}(L)$. Deriving the first order condition immediately yields $L^{-P}=(1-\gamma(1-\lambda)) / \lambda \theta$. Plugging this expression in (18) and solving for $B^{\text {min }}$ leads to

$$
B^{\min }=\frac{1}{\theta} \sqrt{\bar{\delta}-\sigma}\left(1+\frac{1-\lambda}{\lambda}(1-\gamma)\right)
$$

Establishing the second part of the proposition is now straightforward. The condition $\lambda \leq(1-\gamma) \sqrt{\bar{\delta}-\sigma} /(1-\gamma \sqrt{\bar{\delta}-\sigma})$ is simply equivalent to $B^{\min } \geq B^{*}$. In this case, we obviously have $p\left(B^{*}\right)=1$. Hence, the Regulator select $B^{V A}=B^{*}$ without any risk of non-compliance.

If $\lambda>(1-\gamma) \sqrt{\bar{\delta}-\sigma} /(1-\gamma \sqrt{\bar{\delta}-\sigma})$, the equilibrium VA involving perfect compliance is $B^{V A}=B^{\mathrm{min}}$ and we should investigate when $W\left(B^{\min }\right)>W\left(L^{*}\right)$. It is convenient to analyze separately the case where the Polluter is more effective in the lobbying game $(\rho<\gamma)$ and where he is not $(\rho>\gamma)$.

\subsubsection{Case 1: $\rho<\gamma$}

Given (14), $\rho<\gamma$ implies that $L^{*}<B^{*}$. As $W$ is strictly increasing below $B^{*}$, the condition $W\left(B^{\text {min }}\right)>W\left(L^{*}\right)$ is the same as $B^{\text {min }}>L^{*}$. Given (14) and (19), this writes $\lambda<(1-\rho) \sqrt{\bar{\delta}-\sigma} /(1-\rho \sqrt{\bar{\delta}-\sigma})$. Note that this condition is compatible with $\lambda>(1-\gamma) \sqrt{\bar{\delta}-\sigma} /(1-\gamma \sqrt{\bar{\delta}-\sigma})$ since $\rho<\gamma$. 
Moreover $(1-\rho) \sqrt{\bar{\delta}-\sigma} /(1-\rho \sqrt{\bar{\delta}-\sigma})$ is increasing with both $\sqrt{\bar{\delta}-\sigma}$ and $\rho$.

\subsubsection{Case 2: $\rho \geq \gamma$}

Contrary to Case $1, W$ is no longer monotonic between $B^{\min }$ and $L^{*}$, implying that the condition $W\left(B^{\min }\right)>W\left(L^{*}\right)$ does not simplify to $B^{\min }>L^{*}$. As $W$ is single-peaked, $W\left(B^{\mathrm{min}}\right)>W\left(L^{*}\right)$ is now equivalent to $B^{\mathrm{min}}>L^{\prime}$ where $L^{\prime}$ is defined by $W\left(L^{\prime}\right)=W\left(L^{*}\right)$ and $L^{\prime}<B^{*}$.

Let us first identify $L^{\prime}$. Developing $W\left(L^{\prime}\right)=W\left(B^{\min }\right)$ and rearranging, we obtain

$$
L^{\prime}-\frac{1}{2} \theta\left(L^{\prime}\right)^{2}-L^{*}+\frac{1}{2} \theta\left(L^{*}\right)^{2}
$$

which is a polynomial of degree 2 . Solving for $L^{\prime}$, the two roots are $(2 / \theta)-L^{*}$ and $L^{*}$. From $L^{\prime}<B^{*}$ obviously follows that $L^{\prime}=(2 / \theta)-L^{*}$.

As a result, $B^{\text {min }}>L^{\prime}$ is equivalent to $B^{\text {min }}>(2 / \theta)-L^{*}$. Substituting (14) and (19) in this inequality and rearranging leads to

$$
-\lambda^{2}(\rho-\gamma+\rho(1-d \gamma))+\lambda(\rho-\gamma+\rho(1-d \gamma)+d \rho-2 d \gamma \rho-1)+d(1-\gamma)(1-\rho)>0
$$

where $d \equiv \sqrt{\bar{\delta}-\sigma}$. For ease of presentation, we rewrite this inequality as follows

$$
-a \lambda^{2}-\lambda(b-a)+c>0
$$

where $a \equiv \rho-\gamma+\rho(1-d \gamma), b \equiv 1-d(\gamma(1-\rho)+\rho)$ and $c \equiv d(1-\gamma)(1-\rho)$. Note that $a, b, c \geq 0$. It is obvious for $a$ and $c$ since $\rho>\gamma$ and $\rho, \gamma, d \leq 1$. As regards $b$, note that it is decreasing with $d$ since $\gamma(1-\rho)+\rho$ is positive. Then, substituting 
$d=1$ in $b$ yields the expression $1-\rho(1-\gamma)$ which is positive. This proves that $b$ is positive for any parameters.

We now solve (20) for $\lambda$. The determinant writes

$$
\Delta=(b-a)^{2}+4 a c
$$

which is obviously positive. As a result, it admits two roots

$$
\begin{aligned}
& \lambda_{1}=\frac{\sqrt{(b-a)^{2}+4 a c}-(b-a)}{2 a} \\
& \lambda_{2}=\frac{-\sqrt{(b-a)^{2}+4 a c}-(b-a)}{2 a}
\end{aligned}
$$

$\lambda_{2}$ is not a feasible solution since $\lambda_{2}<0$. By contrast $\lambda_{1}>0$. But we need to check whether $\lambda_{1} \leq 1$. Substituting $b=1-d(\gamma(1-\rho)+\rho), c=d(1-\gamma)(1-\rho)$, $d=\sqrt{\bar{\delta}-\sigma}$ and rearranging yields $1-\sqrt{\bar{\delta}-\sigma} \geq 0$ which is satisfied.

The left-hand side of $(20)$ is positive when $\lambda=0$, meaning that $W\left(B^{\min }\right)>$ $W\left(L^{*}\right)$ when $\lambda<\lambda_{1}$. Finally, simulations available upon request show that $\lambda<\lambda_{1}$ is compatible with $\lambda \geq(1-\gamma) \sqrt{\bar{\delta}-\sigma} /(1-\gamma \sqrt{\bar{\delta}-\sigma})$.

\section{References}

[1] G.S. Amacher, A. Malik, Bargaining in environmental regulation and the ideal regulator, J. Environ. Econom. Management 30 (1996) 233-53.

[2] C. Arguedas, Bargaining in environmental regulation revisited, J. Environ. Econom. Management 50 (2005) 422-433. 
[3] S. Arora, S. Gangopadhyay, Towards a theoretical model of voluntary overcompliance, J. Econ. Behav. Organ. 28 (1995) 289-309.

[4] M. Glachant, Voluntary agreements in a rent seeking environment, in: E. Croci (Ed.) Handbook on Environmental Voluntary Agreements, Kluwer Academic Publishers, Dordrecht, Holland, 2005, pp. 49-66.

[5] G.M. Grossman, E. Helpman, Special Interest Politics, MIT Press, Cambridge, 2001.

[6] M. Khanna, The 33/50 voluntary program, in: R.D. Morgenstern and W.A. Pizer (eds) Reality Check: The Nature and Performance of Voluntary Environmental Programs in the US, Europe, and Japan, RFF Press, Washington D.C., 2007, pp. 15-42.

[7] T.P. Lyon, J.W. Maxwell, Self-regulation, taxation and public voluntary environmental agreements, J. Pub. Econ. 87 (2003) 1453-86.

[8] T.P. Lyon, J.W. Maxwell, Corporate Environmentalism and Public Policy, Cambridge University Press, 2004.

[9] P. Manzini and M. Mariotti, A bargaining model of voluntary environmental agreements, J. Pub. Econ. 87 (2003) 2725-2736.

[10] J.W. Maxwell, T.P. Lyon, S.C. Hackett, Self regulation and social welfare: the political economy of corporate environmentalism, J. Law Econ. 43 (2000) 583-617. 
[11] R.D. Morgenstern, W.A. Pizer (eds), Reality Check: The Nature and Performance of Voluntary Environmental Programs in the US, Europe, and Japan, RFF Press, Washington D.C., 2007.

[12] A. Muthoo, Bargaining Theory with Applications, Cambridge University Press, 1999.

[13] OECD, Voluntary Approaches for Environmental Policy: Effectiveness, Efficiency and Usage in Policy Mixes, OECD, Paris, 2003.

[14] K. Segerson, T.J. Miceli, Voluntary environmental agreements: good or bad news for environmental protection?, J. Environ. Econom. Management 36 (1998) 109-130.

\section{Notes}

${ }^{1} \mathrm{~A}$ comprehensive discussion of the literature on VAs is available in Lyon and Maxwell [1].

${ }^{2} \mathrm{~A}$ few papers have analyzed VAs obtained in exchange of a subsidy (see for instance [7]) or VAs driven by demand considerations [3].

${ }^{3}$ Some VAs are proposed in the absence of legislative threats. For instance, U.S. climate change programs such as Climate Wise are used by EPA whereas the agency had no statutory authority to take formal regulatory actions in this field. In these cases, companies join public voluntary agreements in order to obtain technical assistance and/or favorable publicity from the government ([13], p 1457). Our paper does not deal with such agreements. 
${ }^{4}$ Put differently, VAs are used in contexts where mandatory intervention is difficult. This is very intuitive. Why would public authorities rely on voluntary actions by polluters if legislation was easy to pass?

${ }^{5}$ Imperfect compliance with mandatory standards is sometimes observed in reality. However, such standards are at least enforceable in contrast with most VAs. Our assumption makes this difference very clear-cut.

${ }^{6} \operatorname{In}(4), x(L)$ is discounted in line with the idea that discovering non-compliance and launching a legislative process takes time. One may rightly argue that the lobby group contributes before legislation is enacted. This could justify the introduction of a specific discount factor for $x(L)$. This alternative assumption would not alter the results qualitatively. It would simply modify the composition of the sanction cost, by giving more weight to $x(L)$ than to $C(L)$.

${ }^{7}$ The uniformity of the distribution simplifies the presentation of the results. The results will be valid with other distributions, assuming the cumulative and density are positive and increasing on the whole interval.

${ }^{8}$ Note that, when the VA involves a sector, discount rates may differ across firms. We assume here that firms have solved their collective action problem. This implies that, inter alia, they have reached a consensus on a collective discount rate.

${ }^{9} \mathrm{~A}$ bargaining outcome is said to be ex post efficient if and only if after all the information is revealed the players' payoffs associated with the bargaining outcome are Pareto-efficient. Payoffs are said to be correlated when the piece of private information (here $\delta$ ) affects both players' payoffs.

${ }^{10}$ Under this assumption, $L=\lambda / \theta, B^{\min }=(1 / \theta) \sqrt{\lambda(\bar{\delta}-\sigma)}, B^{\max }=(1 / \theta) \sqrt{\lambda(\bar{\delta}+\sigma)}$ and the compliance probability of Eq. $(7)$ is $\frac{1}{2 \sigma}\left(\bar{\delta}+\sigma-\frac{(\theta B)^{2}}{\lambda}\right)$ for any $B \in\left[B^{\min }, B^{\max }\right]$.

${ }^{11}$ Common to all types is the fact that the participation constraints of the pol- 
luter and of the regulator are jointly satisfied. This is so even for self regulation where the Regulator is seemingly absent. It necessarily agrees with the unilateral voluntary commitment, albeit implicitly, because, otherwise, it would implement the threat.

${ }^{12}$ If one does not need to compute equilibrium contributions, assuming differentiability of contribution functions is sufficient to derive the political equilibrium. Differentiability implies that contributions are locally compensating in equilibrium. ${ }^{13}$ As done by Lyon and Maxwell [13] for instance. 


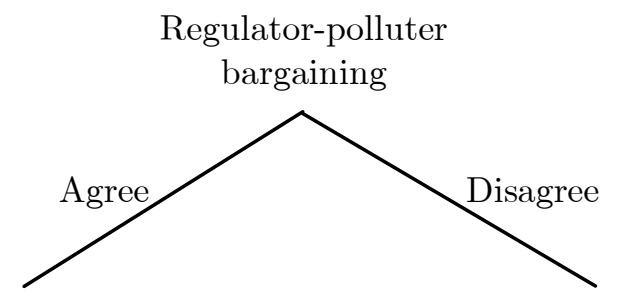

Implement $B$

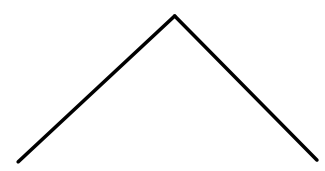

The polluter complies

The polluter does not comply

The regulator initiates the legislative process<smiles>C=C</smiles>

The polluter selects $x$

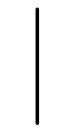

The Congress enacts a quota $L$
The regulator initiates the legislative process

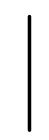

The polluter selects $x$

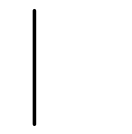

The Congress enacts a quota $L$

Figure 1: Decision tree of the VA policy game 


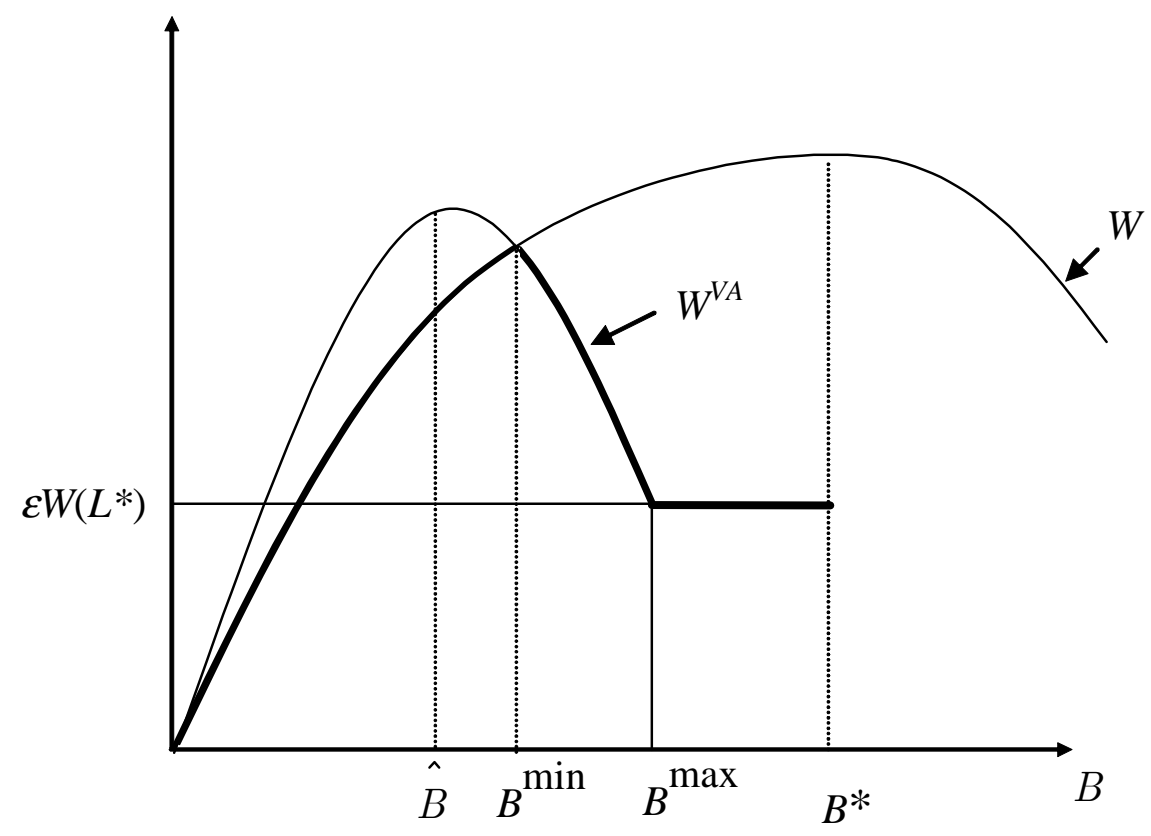

Figure 2a: $W^{V A}$ (in bold) and $W$ if $W\left(B^{\max }\right) \geq \varepsilon W(L)$ and $F^{\prime}\left(B^{\min }\right) \leq 0$

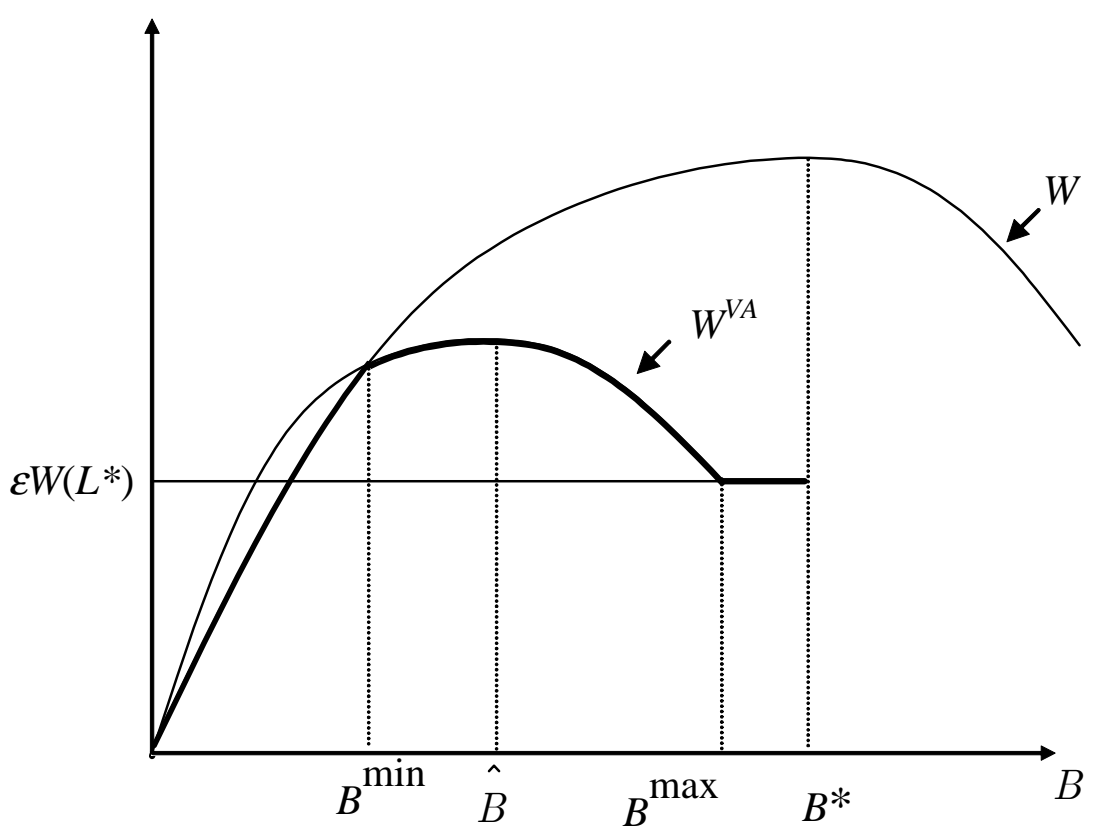

Figure 2b: $W^{V A}$ (in bold) and $W$ if $W\left(B^{\max }\right) \geq \varepsilon W(L)$ and $F^{\prime}\left(B^{\min }\right)>0$ 


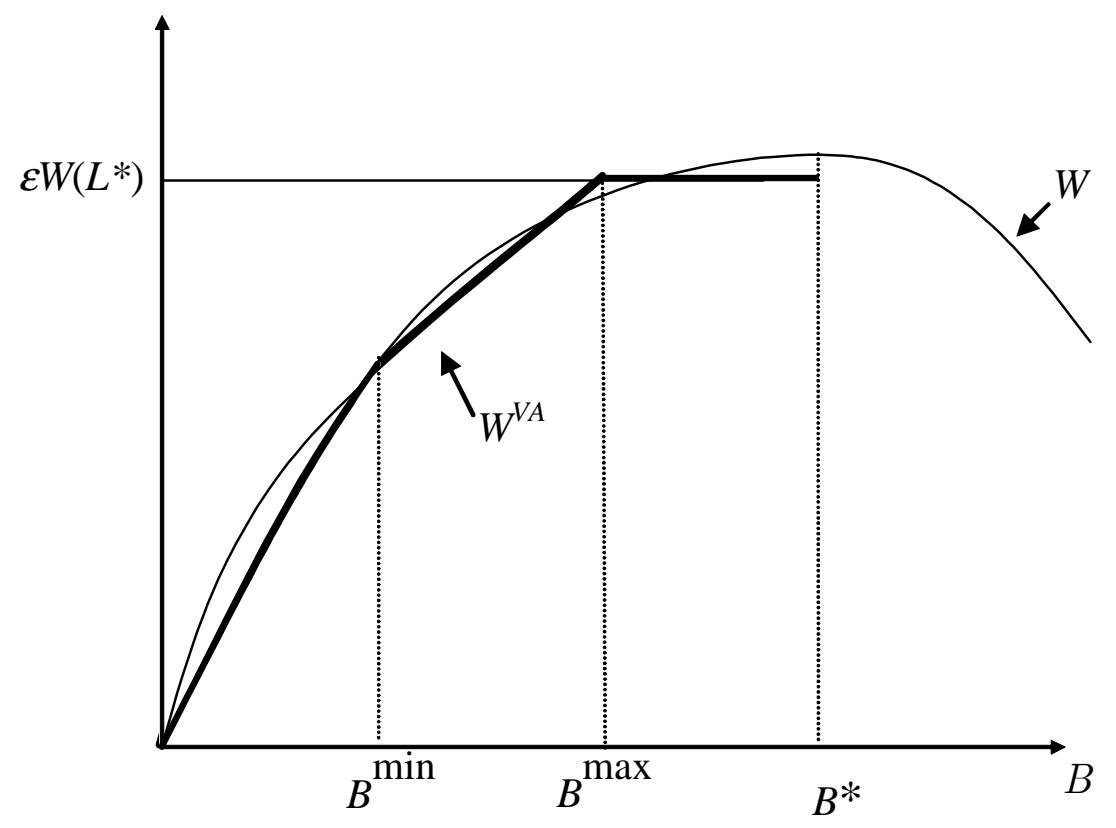

Figure 2c: $W^{V A}$ (in bold) and $W$ if $W\left(B^{\max }\right)<\varepsilon W(L)$ 